Review article

\title{
Variability in non-invasive brain stimulation studies: Reasons and results
}

\author{
Andrea Guerra $^{\mathrm{a}}$, Virginia López-Alonso ${ }^{\mathrm{b}}$, Binith Cheeran ${ }^{\mathrm{c}, \mathrm{d}, *}$, Antonio Suppa ${ }^{\mathrm{a}, \mathrm{e}}$ \\ a Department of Human Neuroscience, Sapienza University of Rome, Viale dell'Università 30, 00185, Rome, Italy \\ b Department of Physical Activity and Sport Sciences, “Centro de Enseñanza Superior Alberta Giménez" Comillas Pontifical University, Costa de Saragossa 16, 07013, \\ Palma, Spain \\ ' St. George's, University of London, London SW17 ORE, UK \\ d The London Clinic, 20 Devonshire Pl, Marylebone, London W1G 6BW, UK \\ e IRCCS Neuromed, Pozzilli, via Atinense 18, 86077, Pozzilli IS, Italy
}

\section{A R T I C L E I N F O}

\section{Keywords:}

Non-invasive brain stimulation

Transcranial magnetic stimulation

Theta burst stimulation

Paired associative stimulation

Transcranial direct current stimulation

\begin{abstract}
A B S T R A C T
Non-invasive brain stimulation techniques (NIBS), such as Theta Burst Stimulation (TBS), Paired Associative Stimulation (PAS) and transcranial Direct Current Stimulation (tDCS), are widely used to probe plasticity in the human motor cortex (M1). Although TBS, PAS and tDCS differ in terms of physiological mechanisms responsible for experimentally-induced cortical plasticity, they all share the ability to elicit long-term potentiation (LTP) and depression (LTD) in M1. However, NIBS techniques are all affected by relevant variability in intra- and intersubject responses. A growing number of factors contributing to NIBS variability have been recently identified and reported. In this review, we have readdressed the issue of variability in human NIBS studies. We have first briefly discussed the physiological mechanisms responsible for TBS, PAS and tDCS-induced cortical plasticity. Then, we have provided statistical measures of intra- and inter-subject variability, as calculated in previous studies. Finally, we have reported in detail known sources of variability by categorizing them into physiological, technical and statistical factors. Improving knowledge about sources of variability could lead to relevant advances in designing new tailored NIBS protocols in physiological and pathological conditions.
\end{abstract}

\section{Introduction}

Over the last two decades, an increasing number of researchers have applied a variety of non-invasive brain stimulation (NIBS) techniques to probe plasticity processes in the human primary motor cortex (M1). In NIBS studies, plasticity commonly refers to changes in cortical excitability, assessed by measures such as motor evoked potential (MEP) amplitudes, outlasting brain stimulation by minutes. Less frequently, interference or improvements in behavioural and motor learning tasks have been used to assess the impact of NIBS protocols. Most NIBS techniques are promising and are met with enthusiasm; the newly-described methods are effective and reliable, but nonetheless are followed by reports of variability in intra-subject and inter-subject response (for a detailed review see [1]). Consequently, the issue of variability has raised concerns about the reliability of NIBS as a therapeutic approach for applications such as neurorehabilitation but also its validity as an experimental tool. We provide an opinionated review with the aim of analysing and discussing critically the issue of variability in NIBS studies. In the first part of this review we describe the physiological mechanisms activated by the currently used NIBS protocols, such as Theta Burst Stimulation (TBS), Paired Associative Stimulation (PAS) and transcranial direct current stimulation (tDCS). We then provide quantitative measures of intra- and inter-subject variability reported in previous studies. Finally, we review specific known factors leading to intra- and inter-subject variability in NIBS.

\section{Common NIBS protocols}

Among NIBS protocols, TBS consists of rhythmic gamma bursts $(50 \mathrm{~Hz})$, repeated at theta frequencies $(5 \mathrm{~Hz})$ at low intensity over M1. The initial report by Huang et al. [2] demonstrated that the intermittent application of TBS (iTBS) could potentiate MEPs, whereas the continuous form (cTBS) could reduce MEP amplitudes for about 30-60 min. The putative mechanism is long-term potentiation (LTP) and long-term depression (LTD)-like plasticity, as human TBS was designed to resemble TBS protocols used to trigger LTP or LTD in brain slices [2,3].

Another NIBS technique widely used in human studies is PAS. PAS combines repetitive electrical nerve stimulation with transcranial magnetic stimulation (TMS) over the contralateral M1 at specific interstimulus intervals (ISIs). PAS at $25 \mathrm{~ms}$ ISI increases MEP amplitudes, whereas PAS at $10 \mathrm{~ms}$ ISI is thought to decrease MEP amplitudes for $30-60 \mathrm{~min}$ [4]. PAS is considered to elicit a Hebbian form of spike-

\footnotetext{
* Corresponding author at: St. George's, University of London, London SW17 0RE, UK.

E-mail address: bcheeran@sgul.ac.uk (B. Cheeran).
} 
timing-dependent plasticity (STDP) in humans [5-7]. PAS may induce LTP/LTD-like phenomena in M1, through repetitive activation of specific sensorimotor circuits within a narrow time window $[4,8]$. Several modified PAS protocols have also been developed: for instance, PAS protocols implying afferent inputs to M1 other than somatosensory (e.g. visual, auditory or nociceptive stimuli, etc.) or protocols consisting of paired TMS pulses delivered over different cortical areas (e.g. corticocortical PAS) [8-11].

Another NIBS protocol currently used in plasticity studies in humans is the transcranial direct current stimulation (tDCS). During tDCS, when the anode is commonly placed over M1 and the cathode over the contralateral frontal pole (anodal tDCS), MEPs tend to increase in amplitude, whereas when polarity is inverted (cathodal tDCS), MEPs tend to decrease in amplitude for $30-60 \mathrm{~min}$. tDCS is thought to mimic protocols of cortical polarisation described in early studies in rats showing that cortical polarisation resulted in a long-term modification of the amplitude of somatosensory evoked potentials elicited by electric stimuli delivered over the skin of the contralateral forepaw [12,13]. Accordingly, tDCS may work through whole-brain polarisation and is believed to induce depolarization or hyperpolarization of the resting membrane potential leading to LTP/LTD-like mechanisms [14]. In particular, tDCS-induced after-effects seem to be mostly related to intracellular calcium dynamics and N-methyl-D-aspartate (NMDA) receptor activity $[1,15,16]$. Gamma-aminobutyric acid (GABA) neurotransmission down-modulation can also play a role $[17,18]$.

\section{Intra- and inter-subject variability in NIBS studies}

Reliability is defined as the extent to which measurements can be replicated. A number of studies have consistently demonstrated that the response to all NIBS protocols are rather variable in healthy humans with a substantial portion of subjects considered as non-responders. We have summarized the previously published data concerning intra-subject and inter-subject variability for TBS, PAS and tDCS protocols used in healthy subjects in Tables 1-3. Studies are arranged by the NIBS technique, and then by protocol (intensity and duration). Several quantitative measures, including Intraclass Correlation and the grand average of the percentage of responders, are provided to evaluate intraand inter-subject variability.

The Intraclass Correlation Coefficient (ICC) is a widely used reliability index in test-retest, intra-rater, and inter-rater reliability analyses. ICC measures are an excellent way to describe test-retest (intra-individual) variability as it reflects both degree of correlation (like Pearson's Correlation) and agreement between measurements (like $t$ tests or Bland Altman plots). Based on the $95 \%$ confident interval of the ICC estimate, values less than 0.5 , between 0.5 and 0.75 , between 0.75 and 0.9 , and higher than 0.90 are indicative of poor, moderate, good, and excellent reliability, respectively.

When considering intra-subject variability in NIBS studies described in Table 1, one might be tempted to estimate the ICC of the most commonly applied tDCS protocol (e.g. $1 \mathrm{~mA}, 10 \mathrm{~min}$ ) to be moderate (0.5-0.75) in the first $30 \mathrm{~min}$ after stimulation. This appears to improve if the duration of stimulation is longer (e.g. $15 \mathrm{~min}$ ), but an increase in stimulation strength over a shorter duration does not appear to improve matters. iTBS and cTBS using standard parameters also show moderate ICC but there are no sham-controlled studies to date. Concerning PAS, a poor ICC has been estimated (Table 1).

When considering inter-subject variability (Tables 2-3), two descriptive statistical approaches are common: arithmetic means (statistical tests such as T-Tests or ANOVA on averages of all measures at individual time points or grand averages of all measures at all time points) and Clustering. The use of clustering has grown in popularity with the recognition that the arithmetic mean of a measure with high response variability between subjects is not a good summary descriptor of the data. Clustering methods like k-means minimize within-cluster variance, thus producing clusters (groups within the larger data set).
The approach taken can affect the interpretation of the data. For example, Lopez-Alonso et al. [19] and Hinder et al. [20] reported results on variability in response to iTBS that support very different conclusions. Hinder et al. [20] reported $73 \%$ of responders (based on grand mean average) while Lopez-Alonso et al. [19] reported $43 \%$ of responders (based on clustering) to the technique.

\section{Factors leading to intra- and inter-subject variability in NIBS studies}

A large number of factors have been identified and reported to explain a variable amount of intra- and inter-subject variability in NIBS studies. We summarize here known factors contributing to intra- and inter-variability by dividing them into three main categories: physiological, technical and statistical aspects.

\subsection{Physiological state}

This category refers to non-modifiable and modifiable factors that influence the physiological state at the time when the NIBS protocol is applied. Most of these physiological factors have been reported in detail in previous consensus papers $[1,3,8,21]$. In brief, non-modifiable factors refer to those subjective characteristics which are immutable for all practical purposes in experimental sessions: age, gender, handedness and genetics. Physiological aging, for instance, has been related to a decline in M1 plasticity [22-24], but differences in response to NIBS have also been reported depending on gender, handedness and, particularly for tDCS, skull conformation and skin condition [25-28]. The most studied genetic factor influencing the direction and amount of response to NIBS protocols has been the Brain-Derived Neurotrophic Factor (BDNF) genotype. Although not consistently replicated [29-31], it has been demonstrated that M1 plasticity is impaired in individuals expressing the Val66Met polymorphism [32-34]. Adversely, carrying specific allelic variants in NMDA receptor subunits enhances the iTBSinduced LTP-like plasticity [35].

When considering modifiable factors of variability in NIBS, intake of medical and non-medical substances is an important consideration. Caffeine, nicotine and alcohol [36-38], as well as Antidepressants, Benzodiazepines and Antiepileptic drugs influence brain excitability and plasticity [39]. Quality of sleep and arousal also modulates NIBS effects: sleep deprivation the night before and reduced alertness or attention during the execution of protocols influence the results [40-42]. Moreover, the state of motor system activation should be considered since prolonged physical activity and/or motor pre-activation prior, during or immediately after NIBS can alter LTP/LTD-like plasticity [43-45]. On a related note, the state of M1 intracortical excitability at the time of applying a NIBS protocol can influence the outcome. The strength of short-interval intracortical inhibition (SICI) at baseline can predict the effectiveness of PAS $[19,46]$. Also, the level of short latency afferent inhibition (SAI) inversely correlates with LTP-like PAS-induced effects, accounting for $\approx 40 \%$ of the inter-individual variability in response [47]. Hormones and their cyclic fluctuations are also well known factors of intra- and inter-subject variability. In particular, female hormones, such as progesterone, and circadian variations of cortisol blood levels modulate cortical excitability [48-50].

\subsection{Technical factors}

A failure of a technology (like a specific NIBS protocol) can be conceived as being due to several factors. Technical factors include those operator-dependent methodological details that need to be carefully controlled while conducting the NIBS experiment. For instance, different elements of M1 are preferentially activated by using different TMS coil orientations and the optimal position for eliciting late I-waves is posterior-to-anterior [51]. Precise and continuous stimulation of the targeted area is obviously needed to induce effective neuromodulation 
Table 1

Intra-subject variability for various NIBS techniques.

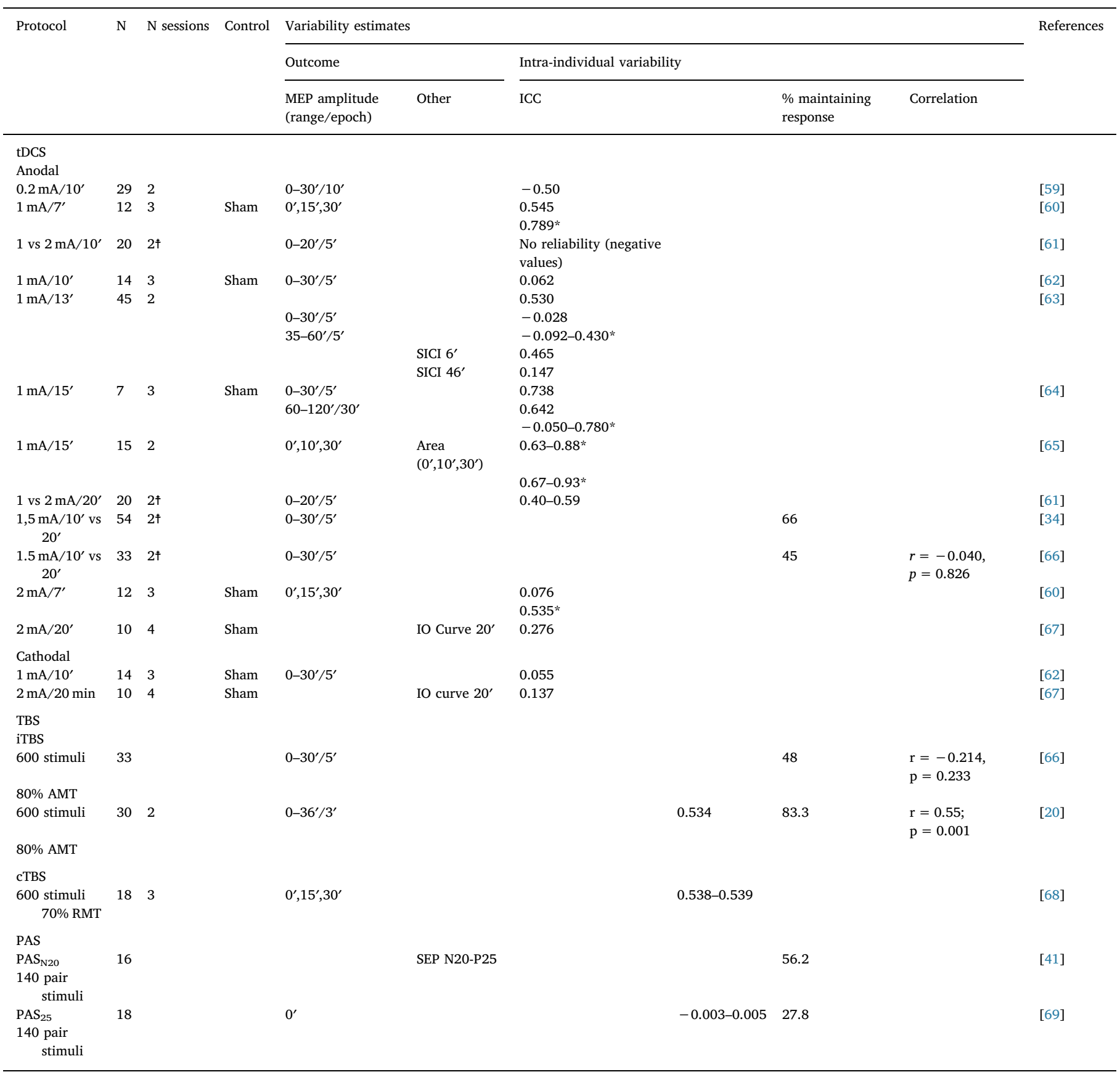

*ICC of single time points (range or best response); fcomparison of sessions with different conditions.

SEP - Somatosensory Evoked Potentials.

[52,53]. Moreover, type (monophasic/biphasic), intensity (low/high), frequency and total number of TMS stimuli significantly affect the direction and amounts of NIBS after-effects [1,8,54]. Regarding tDCS, differences in electrodes size, intensity used, montages, targeted area and duration of stimulation can be responsible of intra- and inter-individual variability. International guidelines are already available for many of these factors (see for example [27]). For others a consensus statement is still lacking (e.g. use of a neuronavigation system to ensure the precise stimulation of the same "spot" throughout the neuromodulation protocol and during the MEPs' serial measurements post-intervention), even though several recommendations for best technical practice have been recently proposed for PAS studies [8]. However, there is little or no consensus on minimum requirements for training and assessment of technical competency, in a field where the majority of literature is produced by graduate or postgraduate students with variable experience, expertise and supervision. Technical factors generally affect both inter- and intra-subject variability, and explicit standards for training as well as consensus guidelines for individual NIBS protocols are necessary for reliable, reproducible results. A final comment involves the consideration that each NIBS protocol can be thought of as a technology. The most obvious factor in this category is an intrinsic lack of efficacy. However, factors other than raw efficacy should be considered to minimize variability. Technology can fail to benefit when it is improperly applied (technical factors) and if a particular technology is difficult to apply, failures are more common. Some factors, such as basing stimulation intensity on a factor open to inter- 
Table 2

Inter-subject variability after Transcranial Direct Current Stimulation.

\begin{tabular}{|c|c|c|c|c|c|c|c|c|}
\hline \multirow[t]{3}{*}{ Protocol } & \multirow[t]{3}{*}{$\mathrm{N}$} & \multirow[t]{3}{*}{ Control } & \multicolumn{5}{|l|}{ Variability estimates } & \multirow[t]{3}{*}{ References } \\
\hline & & & \multicolumn{2}{|l|}{ Outcome } & \multicolumn{3}{|l|}{$\%$ of Responders } & \\
\hline & & & MEP amplitude (range/epochs) & Other & Grand Average & Clustering & Other measures & \\
\hline \multicolumn{9}{|l|}{ tDCS } \\
\hline \multicolumn{9}{|l|}{ Anodal } \\
\hline $0.2 \mathrm{~mA} / 10^{\prime}$ & 29 & & $0-30^{\prime} / 10^{\prime}$ & & $34.5^{* *}$ & 41 & & [59] \\
\hline $0.5 \mathrm{~mA} / 10^{\prime}$ & 29 & & $0-30^{\prime} / 10^{\prime}$ & & $17.2-24.1^{* *}$ & & & [59] \\
\hline $0.5 \mathrm{~mA} / 15^{\prime}$ & 20 & Sham & $0-30^{\prime} / 5^{\prime}$ & & 75 & & & [64] \\
\hline $1 \mathrm{~mA} / 7^{\prime}$ & 29 & & $0-30^{\prime} / 5^{\prime}$ & & & & 79.3* (single best timepoint MEP) & {$[70]$} \\
\hline $1 \mathrm{~mA} / 7^{\prime}$ & 12 & Sham & $0^{\prime}, 15^{\prime}, 30^{\prime}$ & & $33.3^{* * *}$ VBTs & 25 & & [60] \\
\hline $1 \mathrm{~mA} / 9^{\prime}$ & 59 & & $0^{\prime}, 5^{\prime}, 10^{\prime}, 20^{\prime}, 30^{\prime}, 40^{\prime}$ & & 61 & $41 \mp$ & & [71] \\
\hline $1 \mathrm{~mA} / 10^{\prime}$ & 29 & & $0-30^{\prime} / 10^{\prime}$ & & $20.7 * *$ & & & [59] \\
\hline $1 \mathrm{~mA} / 10^{\prime}$ & 20 & & $0-20^{\prime} / 5^{\prime}$ & & $35 \$$ & & & [61] \\
\hline \multirow[t]{2}{*}{$1 \mathrm{~mA} / 10^{\prime}$} & 14 & Sham & $0-30^{\prime} / 5^{\prime}$ & & $57.1-64.3$ & & & [62] \\
\hline & & & & & $28.6-42.8^{* *}$ & & & \\
\hline $1 \mathrm{~mA} / 13^{\prime}$ & 56 & & $0-60^{\prime} / 5^{\prime}$ & SICI $\left(6^{\prime}, 46^{\prime}\right)$ & 50 & 45 & 56.3 & [19] \\
\hline $1 \mathrm{~mA} / 13^{\prime}$ & 45 & & $0-60^{\prime} / 5^{\prime}$ & & $51.1-64.4$ & & & [63] \\
\hline \multirow[t]{3}{*}{$1 \mathrm{~mA} / 13^{\prime}$} & 30 & & $0^{\prime}, 5^{\prime}, 10^{\prime}, 20^{\prime}, 30^{\prime}$ & & 66 & & & {$[72]$} \\
\hline & & & & & $55^{*}$ & & & \\
\hline & & & & & $21^{* * * *}$ & & & \\
\hline $1 \mathrm{~mA} / 15^{\prime}$ & 20 & Sham & $0-30^{\prime} / 5^{\prime}$ & & 90 & & & [64] \\
\hline $1 \mathrm{~mA} / 20^{\prime}$ & 26 & Sham & $0^{\prime}, 5^{\prime}$ & & 35.7 & & & [73] \\
\hline $1 \mathrm{~mA} / 20^{\prime}$ & 20 & & $0-20^{\prime} / 5^{\prime}$ & & $35 \$$ & & & [61] \\
\hline $1,5 \mathrm{~mA} / 10^{\prime}$ & 54 & & $0-30^{\prime} / 5^{\prime}$ & & 64 & 42 & & [34] \\
\hline $1.5 \mathrm{~mA} / 10^{\prime}$ & 33 & & $0-30^{\prime} / 5^{\prime}$ & & $55^{*}$ & & & [66] \\
\hline $1.5 \mathrm{~mA} / 15^{\prime}$ & 20 & Sham & $0-30^{\prime} / 5^{\prime}$ & & 75 & & & [64] \\
\hline $1.5 \mathrm{~mA} / 20^{\prime}$ & 54 & & $0-30^{\prime} / 5^{\prime}$ & & 62 & 42 & & [34] \\
\hline $1.5 \mathrm{~mA} / 20^{\prime}$ & 33 & & $0-30^{\prime} / 5^{\prime}$ & & $52^{*}$ & & & [66] \\
\hline $2 \mathrm{~mA} / 7^{\prime}$ & 12 & Sham & $0^{\prime}, 15^{\prime}, 30^{\prime}$ & & $52.8^{* * *}$ VBTs & 47.2 & & [60] \\
\hline $2 \mathrm{~mA} / 10^{\prime}$ & 53 & & $0-30^{\prime} / 5^{\prime}$ & & 74 & 47.2 & & [74] \\
\hline $2 \mathrm{~mA} / 10^{\prime}$ & 29 & & $0-30^{\prime} / 10^{\prime}$ & & $38^{* *}$ & 21 & & [59] \\
\hline $2 \mathrm{~mA} / 10^{\prime}$ & 20 & & $0-20^{\prime} / 5^{\prime}$ & & $20 \$$ & & & [61] \\
\hline $2 \mathrm{~mA} / 15^{\prime}$ & 20 & Sham & $0-30^{\prime} / 5^{\prime}$ & & 85 & & & [64] \\
\hline $2 \mathrm{~mA} / 20^{\prime}$ & 20 & & $0-20^{\prime} / 5^{\prime}$ & & $35 \$$ & & & [61] \\
\hline \multicolumn{9}{|l|}{ Cathodal } \\
\hline $1 \mathrm{~mA} / 9^{\prime}$ & 59 & & $0^{\prime}, 5^{\prime}, 10^{\prime}, 20^{\prime}, 30^{\prime}, 40^{\prime}$ & & 53 & $49 \dagger$ & & [71] \\
\hline \multirow[t]{2}{*}{$1 \mathrm{~mA} / 10^{\prime}$} & 14 & Sham & $0-30^{\prime} / 5^{\prime}$ & & $21.4-42.9$ & & & [62] \\
\hline & & & & & $0-28.6^{* *}$ & & & \\
\hline $2 \mathrm{~mA} / 10^{\prime}$ & 53 & & $0-30^{\prime} / 5^{\prime}$ & & 41 & 52.8 & & [74] \\
\hline $2 \mathrm{~mA} / 20^{\prime}$ & 20 & & $10^{\prime}, 20^{\prime}$ & SLP ${ }_{\text {ac }} 20^{\prime}, 40^{\prime}$ & $42^{* *}$ & & 40 & [75] \\
\hline
\end{tabular}

SICI - Short-interval intracortical inhibition; SLP ${ }_{\mathrm{ac}}$ - Silent-period latency during active contraction; VBTs - Sham Variability-Based Threshold.

Grand average cut-off is $100 \%$ unless $* 110, * * 120, * * * 130, * * * * 150$, \$Other cut-offs.

Clusters $\rightarrow$ 2Steps unless † Agglomerative hierarchical clustering or other clustering methods.

observer variability such as Active Motor Threshold, may be intrinsic to a particular NIBS protocol.

\subsection{Statistical aspects}

The very first step of any study should be to formulate a priori hypothesis and, only after that, to design the experiment [55]. A publish-or-perish mentality and editorial preference for 'positive' studies can result in data mining and publication bias. It is also important to state that most NIBS studies have been conducted on relatively low numbers of subjects (see Tables 1-3). Using an inadequate sample size is a relevant methodological issue contributing to variability and affecting the reliability of NIBS studies. Finally, the variance can result not just from the NIBS intervention itself, but also the assay utilized to assess outcomes [55]. The most common assay is the MEP, which is collected and averaged in most TMS studies in blocks of 15-30 MEPs per time point (see for example $[20,56,57]$ ). Accuracy and reliability of TMS measures are definitely low when averaging a reduced number of trials [58]. Moreover, time dynamics of neuromodulatory interventions cannot be adequately described when testing a low number of time points. A final consideration concerns the statistical analysis used for evaluating NIBS after-effects. It should be taken into account that the use of parametric tests is allowed only when the assumptions of normality of the distributions are satisfied. Using parametric tests for evaluating data that is not normally-distributed might influence the reliability of NIBS studies [55]. The same effect can be caused by arbitrary outlier rejection and use of inappropriate summary statistics.

\section{Conclusions}

In the present review, we have readdressed the issue of intra- and inter-subjects variability and tabulated the pertinent published literature for the commonest NIBS protocols. We have also summarized the known factors contributing to variability in human studies. At the very least, this should confirm the growing consensus that variability in response to NIBS is a consistent and significant research issue. However, although a number of factors contributing to intra- and inter-subject variability have been identified, their relative importance is uncertain. Moreover, it is unlikely that we have already discovered all possible sources of variability in response to NIBS. Hence, further studies aimed to identify response modifiers and to quantify their relative importance are warranted. While the issue of variance in outcomes of NIBS protocols continues to attract criticism about the relevance, reliability and the science of NIBS from some quarters, we believe they should be considered an opportunity. A more detailed understanding of the sources of variability is fundamental to understand the basis for altered 
Table 3

Inter-subject variability after Theta Burst Stimulation and Paired Associative Stimulation.

\begin{tabular}{|c|c|c|c|c|c|c|c|c|}
\hline \multirow[t]{3}{*}{ Protocol } & \multirow[t]{3}{*}{$\mathrm{N}$} & \multirow[t]{3}{*}{ Control } & \multicolumn{5}{|l|}{ Variability estimates } & \multirow[t]{3}{*}{ References } \\
\hline & & & \multicolumn{2}{|l|}{ Outcome } & \multicolumn{3}{|l|}{$\%$ of Responders } & \\
\hline & & & MEP amplitude (range/epoch) & Other & Grand Average & Clustering & Other measures & \\
\hline \multicolumn{9}{|l|}{ TBS } \\
\hline \multicolumn{9}{|l|}{ iTBS } \\
\hline 300 stimuli @ 80\% AMT & 33 & & $0-30^{\prime} / 5^{\prime}$ & & $58^{*}$ & No Cluster & & {$[66]$} \\
\hline 600 stimuli @ 80\% AMT & 56 & & $0-60^{\prime} / 5^{\prime}$ & SICI $\left(6^{\prime}, 46^{\prime}\right)$ & 46.4 & 43 & 54.6 & [19] \\
\hline 600 stimuli @ 80\% AMT & 52 & & $0-30^{\prime} / 5^{\prime}$ & & 52 & & & {$[56]$} \\
\hline 600 stimuli @ 70\% RMT & 40 & & $5^{\prime}, 15^{\prime}, 25^{\prime}$ & & 47.5 & & & [76] \\
\hline 600 stimuli @ 80\% AMT & 38 & & $0^{\prime}, 5^{\prime}, 10^{\prime}, 20^{\prime}$ & & $56-65$ & & & {$[77]$} \\
\hline 600 stimuli @ 80\% AMT & 33 & & $0-30^{\prime} / 5^{\prime}$ & & $55^{*}$ & 52 & & {$[66]$} \\
\hline \multirow[t]{2}{*}{600 stimuli @ 80\% AMT } & 30 & & $0-36^{\prime} / 3^{\prime}$ & & 73 & & & {$[20]$} \\
\hline & & & & & $60^{*}$ & & & \\
\hline 600 stimuli @ 80\% AMT & 16 & Sham & $0^{\prime}$ & & $44^{*}$ & & & {$[78]$} \\
\hline 600 stimuli @ 80\% AMT & 16 & & $0-60^{\prime} / 10^{\prime}$ & & 62.5 & & & [79] \\
\hline \multicolumn{9}{|l|}{ cTBS } \\
\hline 600 stimuli @ 80\% AMT & 52 & & $0-30^{\prime} / 5^{\prime}$ & & 42 & & & {$[56]$} \\
\hline 600 stimuli@ @70\% RMT & 36 & & $0-30^{\prime} / 5^{\prime}$ & & $72^{* *}$ & & & [44] \\
\hline 600 stimuli @ 70\% RMT & 34 & & $0^{\prime} / 20^{\prime}$ & & $35.3-47.1$ & & & [80] \\
\hline 600 stimuli @ 80\% AMT & 31 & & $5^{\prime}, 10^{\prime}, 20^{\prime}, 30^{\prime}, 40^{\prime}, 50^{\prime}$ & & 19.3 & & & [81] \\
\hline 600 stimuli @ 80\% AMT & 24 & & $10^{\prime}, 25^{\prime}, 40^{\prime}$ & & 62.5 & & & [82] \\
\hline 600 stimuli @ 80\% AMT & 21 & & $5^{\prime}, 10^{\prime}, 15^{\prime}, 20^{\prime}, 30^{\prime}, 40^{\prime}, 50^{\prime}$ & & & $57.1 \dagger$ & & [83] \\
\hline 600 stimuli @ 70\% RMT & 18 & & $0^{\prime}, 15^{\prime}, 30^{\prime}$ & & $61-78$ & & & {$[68]$} \\
\hline 600 stimuli @ 70\% RMT & 10 & & $0-30^{\prime} / 5^{\prime}$ & & $70 * *$ & & & {$[44]$} \\
\hline 600 stimuli @ 80\% RMT & 8 & & $0^{\prime}, 20^{\prime}$ & & $38-75$ & & & [84] \\
\hline \multicolumn{9}{|l|}{ PAS } \\
\hline Different protocols & 190 & & & & 53 & & & [85] \\
\hline $\begin{array}{l}\mathrm{PAS}_{25} \\
200 \text { paired stimuli } \\
\text { Ulnar nerve }\end{array}$ & 56 & & $0-60^{\prime} / 5^{\prime}$ & SICI $\left(6^{\prime}, 46^{\prime}\right)$ & 39 & 53.6 & 46.5 & [19] \\
\hline $\begin{array}{l}\mathrm{PAS}_{25} \\
180 \text { paired stimuli } \\
\text { Median nerve }\end{array}$ & 37 & & $1^{\prime}, 8^{\prime}, 15^{\prime}$ & & 63 & & & [42] \\
\hline $\mathrm{PAS}_{25}$ & 30 & & $0^{\prime}, 5^{\prime}, 10^{\prime}, 20^{\prime}, 30^{\prime}$ & & 79 & & & {$[72]$} \\
\hline 180 paired stimuli & & & & & $72^{*}$ & & & \\
\hline Ulnar nerve & & & & & $47^{* * * *}$ & & & \\
\hline $\begin{array}{l}\mathrm{PAS}_{25} \\
200 \text { paired stimuli } \\
\text { Median nerve }\end{array}$ & 18 & & & & 63 & & & [46] \\
\hline $\begin{array}{l}\mathrm{PAS}_{25} \\
200 \text { paired stimuli } \\
\text { Ulnar nerve }\end{array}$ & 16 & & $0-60^{\prime} / 10^{\prime}$ & & 75 & & & [79] \\
\hline $\begin{array}{l}\mathrm{PAS}_{\mathrm{N} 20} \\
140 \text { paired stimuli } \\
\text { Median nerve }\end{array}$ & 16 & & & $\begin{array}{l}\text { Somatosensory Evoked Potentials } \\
\text { N20-P25 }\end{array}$ & & & 56.2 & [41] \\
\hline $\begin{array}{l}\mathrm{PAS}_{20} \\
225 \text { paired stimuli } \\
\text { Median nerve }\end{array}$ & 14 & & $0-30^{\prime} / 5^{\prime}$ & & 52 & & & [86] \\
\hline
\end{tabular}

Grand average cut-off is $100 \%$ unless $* 110, * * 120, * * * 130, * * * * 150$.

Clusters $\rightarrow 2$ Steps unless $\uparrow$ Agglomerative hierarchical clustering or other clustering methods.

response to NIBS in several neurological disorders (e.g. [3,8]). More importantly, a better understanding of sources of variability would also allow the design of new tailored interventions making the neuromodulation strategies as effective as possible.

\section{References}

[1] Y.Z. Huang, M.K. Lu, A. Antal, J. Classen, M. Nitsche, U. Ziemann, M. Ridding, M. Hamada, Y. Ugawa, S. Jaberzadeh, A. Suppa, W. Paulus, J.C. Rothwell, Plasticity induced by non-invasive transcranial brain stimulation: a critical position paper, Clin. Neurophysiol. 128 (2017) 2318-2329.

[2] Y.Z. Huang, M.J. Edwards, E. Rounis, K.P. Bhatia, J.C. Rothwell, Theta burst stimulation of the human motor cortex, Neuron 45 (2005) 201-206.

[3] A. Suppa, Y.Z. Huang, K. Funke, M.C. Ridding, B. Cheeran, V. Di Lazzaro, U. Ziemann, J.C. Rothwell, Ten years of theta burst stimulation in humans: established knowledge, unknowns and prospects, Brain Stimul. 9 (2016) 323-335.

[4] K. Stefan, E. Kunesch, L.G. Cohen, R. Benecke, J. Classen, Induction of plasticity in the human motor cortex by paired associative stimulation, Brain 123 (2000) 572-584.

[5] D.O. Hebb, The Organization of Behavior, Wiley \& Sons, New York, 1949.
[6] Y. Dan, M.M. Poo, Spike timing-dependent plasticity of neural circuits, Neuron 44 (2004) 23-30.

[7] N. Caporale, Y. Dan, Spike timing-dependent plasticity: a Hebbian learning rule, Annu. Rev. Neurosci. 31 (2008) 25-46.

[8] A. Suppa, A. Quartarone, H. Siebner, R. Cheng, V. Di Lazzaro, P. Del Giudice, W. Paulus, J.C. Rothwell, U. Ziemannm, J. Classen, The associative brain at work: evidence from paired associative stimulation studies in humans, Clin. Neurophysiol. 128 (2017) 2140-2164.

[9] G. Koch, V. Ponzo, F. Di Lorenzo, C. Caltagirone, D. Veniero, Hebbian and antiHebbian spike-timing-dependent plasticity of human cortico-cortical connections, J, Neurosci. 33 (2013) 9725-9733.

[10] D. Veniero, V. Ponzo, G. Koch, Paired associative stimulation enforces the communication between interconnected areas, J. Neurosci. 33 (2013) 13773-13783.

[11] E.P. Casula, M.C. Pellicciari, S. Picazio, C. Caltagirone, G. Koch, Spike-timing-dependent plasticity in the human dorso-lateral prefrontal cortex, Neuroimage 143 (2016) 204-213.

[12] G.H. Bishop, J.L. O'Leary, The effects of polarizing currents on cell potentials and their significance in the interpretation of central nervous system activity, Electroencephalogr. Clin. Neurophysiol. 2 (1950) 401-416.

[13] L.J. Bindman, O.C. Lippold, J.W. Redfearn, The action of brief polarizing currents on the cerebral cortex of the rat (1) during current flow and (2) in the production of long-lasting after-effects, J. Physiol. 172 (1964) 369-382. 
[14] M.A. Nitsche, W. Paulus, Excitability changes induced in the human motor cortex by weak transcranial direct current stimulation, J. Physiol. 527 (2000) 633-639.

[15] M.A. Nitsche, K. Fricke, U. Henschke, A. Schlitterlau, D. Liebetanz, N. Lang, S. Henning, F. Tergau, W. Paulus, Pharmacological modulation of cortical excitability shifts induced by transcranial direct current stimulation in humans, J. Physiol. 553 (2003) 293-301.

[16] H. Monai, M. Ohkura, M. Tanaka, Y. Oe, A. Konno, H. Hirai, K. Mikoshiba, S. Itohara, J. Nakai, Y. Iwai, H. Hirase, Calcium imaging reveals glial involvement in transcranial direct current stimulation-induced plasticity in mouse brain, Nat. Commun. 7 (2016) 11100.

[17] C.J. Stagg, J.G. Best, M.C. Stephenson, J. O'Shea, M. Wylezinska, Z.T. Kincses, P.G. Morris, P.M. Matthews, H. Johansen-Berg, Polarity sensitive modulation of cortical neurotransmitters by transcranial stimulation, J. Neurosci. 29 (2009) 5202-5206.

[18] S. Kim, M.C. Stephenson, P.G. Morris, S.R. Jackson, TDCS-induced alterations in GABA concentration within primary motor cortex predict motor learning and motor memory: a $7 \mathrm{~T}$ magnetic resonance spectroscopy study, Neuroimage 99 (2014) 237-243.

[19] V. López-Alonso, B. Cheeran, D. Río-Rodríguez, M. Fernández-del-Olmo, Interindividual variability in response to non-invasive brain stimulation paradigms, Brain Stimul. 7 (2014) 372-380.

[20] M.R. Hinder, E.L. Goss, H. Fujiyama, A.J. Canty, M.I. Garry, J. Rodger, J.J. Summers, Inter- and intra-individual variability following intermittent theta burst stimulation: implications for rehabilitation and recovery, Brain Stimul. 7 (2014) 365-371.

[21] M.C. Ridding, U. Ziemann, Determinants of the induction of cortical plasticity by non-invasive brain stimulation in healthy subjects, J. Physiol. 588 (2010) 2291-2304.

[22] J.F. Müller-Dahlhaus, Y. Orekhov, Y. Liu, U. Ziemann, Interindividual variability and age-dependency of motor cortical plasticity induced by paired associative stimulation, Exp. Brain Res. 187 (2008) 467-475.

[23] F. Tecchio, F. Zappasodi, P. Pasqualetti, L. De Gennaro, M.C. Pellicciari, M. Ercolani, R. Squitti, P.M. Rossini, Age dependence of primary motor cortex plasticity induced by paired associative stimulation, Clin. Neurophysiol. 119 (2008) 675-682.

[24] S. Bashir, J.M. Perez, J.C. Horvath, C. Pena-Gomez, M. Vernet, A. Capia, M. AlonsoAlonso, A. Pascual-Leone, Differential effects of motor cortical excitability and plasticity in young and old individuals: a Transcranial Magnetic Stimulation (TMS) study, Front Aging Neurosci. 6 (2014) 111.

[25] J. Cirillo, N.C. Rogasch, J.G. Semmler, Hemispheric differences in use-dependent corticomotor plasticity in young and old adults, Exp. Brain Res. 205 (2010) 57-68.

[26] J.C. Horvath, O. Carter, J.D. Forte, Transcranial direct current stimulation: five important issues we aren't discussing (but probably should be), Front Syst. Neurosci. 8 (2014) 2.

[27] J.P. Lefaucheur, A. Antal, S.S. Ayache, D.H. Benninger, J. Brunelin, F. Cogiamanian, M. Cotelli, D. De Ridder, R. Ferrucci, B. Langguth, P. Marangolo, V. Mylius, M.A. Nitsche, F. Padberg, U. Palm, E. Poulet, A. Priori, S. Rossi, M. Schecklmann, S. Vanneste, U. Ziemann, L. Garcia-Larrea, W. Paulus, Evidence-based guidelines on the therapeutic use of transcranial direct current stimulation (tDCS), Clin. Neurophysiol. 128 (2017) 56-92.

[28] L.M. Rogers, Y.Y. Dhaher, Female sex hormones modulate the response to lowfrequency rTMS in the human motor cortex, Brain Stimul. 10 (2017) 850-852.

[29] P. Li Voti, A. Conte, A. Suppa, E. Iezzi, M. Bologna, M.S. Aniello, G. Defazio, J.C. Rothwell, A. Berardelli, Correlation between cortical plasticity, motor learning and BDNF genotype in healthy subjects, Exp. Brain Res. 212 (2011) 91-99.

[30] C. Mastroeni, T.O. Bergmann, V. Rizzo, C. Ritter, C. Klein, I. Pohlmann, N. Brueggemann, A. Quartarone, H.R. Siebner, Brain-derived neurotrophic factor-a major player in stimulation-induced homeostatic metaplasticity of human motor cortex? PLoS One 8 (2013) e57957.

[31] A. Suppa, B. Cheeran, Further insights into the effect of BDNF genotype on noninvasive brain stimulation, Clin. Neurophysiol. 126 (2015) 1281-1283.

[32] B. Cheeran, P. Talelli, F. Mori, G. Koch, A. Suppa, M. Edwards, H. Houlden, K. Bhatia, R. Greenwood, J.C. Rothwell, A common polymorphism in the brainderived neurotrophic factor gene (BDNF) modulates human cortical plasticity and the response to rTMS, J. Physiol. 586 (2008) 5717-5725.

[33] A. Antal, L. Chaieb, V. Moliadze, K. Monte-Silva, C. Poreisz N. Thirugnanasambandam, M.A. Nitsche, M. Shoukier, H. Ludwig, W. Paulus, Brainderived neurotrophic factor (BDNF) gene polymorphisms shape cortical plasticity in humans, Brain Stimul. 3 (2010) 230-237.

[34] R. Puri, M.R. Hinder, H. Fujiyama, R. Gomez, R.G. Carson, J.J. Summers, Durationdependent effects of the BDNF Val66Met polymorphism on anodal tDCS induced motor cortex plasticity in older adults: a group and individual perspective, Front. Aging Neurosci. 7 (2015) 107.

[35] F. Mori, M. Ribolsi, H. Kusayanagi, A. Siracusano, V. Mantovani, E. Marasco, G. Bernardi, D. Centonze, Genetic variants of the NMDA receptor influence cortical excitability and plasticity in humans, J. Neurophysiol. 106 (2011) 1637-1643.

[36] M. Specterman, A. Bhuiya, A. Kuppuswamy, P.H. Strutton, M. Catley, N.J. Davey, The effect of an energy drink containing glucose and caffeine on human corticospinal excitability, Physiol. Behav. 83 (2005) 723-728.

[37] J. Grundey, N. Thirugnanasambandam, K. Kaminsky, A. Drees, A.C. Skwirba, N. Lang, W. Paulus, M.A. Nitsche, Rapid effect of nicotine intake on neuroplasticity in non-smoking humans, Front. Pharmacol. 3 (2012) 186.

[38] C. Lücke, T. Heidegger, M. Röhner, S.W. Toennes, L. Krivanekova, F. MüllerDahlhaus, U. Ziemann, Deleterious effects of a low amount of ethanol on LTP-like plasticity in human cortex, Neuropsychopharmacology 39 (2014) 1508-1518.

[39] U. Ziemann, J. Reis, P. Schwenkreis, M. Rosanova, A. Strafella, R. Badawy,
F. Müller-Dahlhaus, TMS and drugs revisited 2014, Clin. Neurophysiol. 126 (2015) 1847-1868.

[40] K. Stefan, M. Wycislo, J. Classen, Modulation of associative human motor cortical plasticity by attention, J. Neurophysiol 92 (2004) 66-72.

[41] M. Gorgoni, F. Ferlazzo, A. D'Atri, G. Lauri, M. Ferrara, P.M. Rossini, L. De Gennaro, The assessment of somatosensory cortex plasticity during sleep deprivation by paired associative stimulation, Arch. Ital. Biol. 153 (2015) 110-123.

[42] S. Klöppel, E. Lauer, J. Peter, L. Minkova, C. Nissen, C. Normann, J. Reis, F. Mainberger, M. Bach, J. Lahr, LTP-like plasticity in the visual system and in the motor system appear related in young and healthy subjects, Front. Hum. Neurosci. 9 (2015) 506.

[43] J.F. Lepage, O. Morin-Moncet, V. Beaulé, L. de Beaumont, F. Champoux, H. Théoret, Occlusion of LTP-like plasticity in human primary motor cortex by action observation, PLoS One 7 (2012) e38754.

[44] M.R. Goldsworthy, F. Müller-Dahlhaus, M.C. Ridding, U. Ziemann, Inter-subject variability of LTD-like plasticity in human motor cortex: a matter of preceding motor activation, Brain Stimul. 7 (2014) 864-870.

[45] C.S. Mang, N.J. Snow, K.L. Campbell, C.J. Ross, L.A. Boyd, A single bout of highintensity aerobic exercise facilitates response to paired associative stimulation and promotes sequence-specific implicit motor learning, J. Appl. Physiol. (1985) 117 (2014) 1325-1336.

[46] N. Murase, B. Cengiz, J.C. Rothwell, Inter-individual variation in the after-effect of paired associative stimulation can be predicted from short-interval intracortical inhibition with the threshold tracking method, Brain Stimul. 8 (2015) 105-113.

[47] R.F. Cash, T. Murakami, R. Chen, G.W. Thickbroom, U. Ziemann, Augmenting plasticity induction in human motor cortex by disinhibition stimulation, Cereb. Cortex 26 (2016) 58-69.

[48] M.J. Smith, J.C. Keel, B.D. Greenberg, L.F. Adams, P.J. Schmidt, D.A. Rubinow, E.M. Wassermann, Menstrual cycle effects on cortical excitability, Neurology 53 (1999) 2069-2072.

[49] M. Inghilleri, A. Conte, A. Curra, V. Frasca, C. Lorenzano, A. Berardelli, Ovarian hormones and cortical excitability. An rTMS study in humans, Clin. Neurophysiol. 115 (2004) 1063-1068.

[50] M.V. Sale, M.C. Ridding, M.A. Nordstrom, Factors influencing the magnitude and reproducibility of corticomotor excitability changes induced by paired associative stimulation, Exp. Brain Res. 181 (2007) 615-626.

[51] V. Di Lazzaro, U. Ziemann, The contribution of transcranial magnetic stimulation in the functional evaluation of microcircuits in human motor cortex, Front. Neural Circuits. 7 (2013) 18.

[52] P. Julkunen, L. Säisänen, N. Danner, E. Niskanen, T. Hukkanen, E. Mervaala, M. Könönen, Comparison of navigated and non-navigated transcranial magnetic stimulation for motor cortex mapping, motor threshold and motor evoked potentials, Neuroimage 44 (2009) 790-795.

[53] M. Cincotta, F. Giovannelli, A. Borgheresi, F. Balestrieri, L. Toscani, G. Zaccara, F. Carducci, M.P. Viggiano, S. Rossi, Optically tracked neuronavigation increases the stability of hand-held focal coil positioning: evidence from transcranial magnetic stimulation-induced electrical field measurements, Brain Stimul. 3 (2010) $119-123$.

[54] K. Nakamura, S.J. Groiss, M. Hamada, H. Enomoto, S. Kadowaki, M. Abe, T. Murakami, W. Wiratman, F. Chang, S. Kobayashi, R. Hanajima, Y. Terao, Y. Ugawa, Variability in response to quadripulse stimulation of the motor cortex, Brain Stimul. 9 (2016) 859-866.

[55] M. Stecker, P. Pasqualetti, R.J. Barry, Z.J. Daskalakis, H.R. Siebner, U. Ziemann, Statistical data analyses for clinical neurophysiology, Clin. Neurophysiol. 128 (2017) 1837-1838.

[56] M. Hamada, N. Murase, A. Hasan, M. Balaratnam, J.C. Rothwell, The role of interneuron networks in driving human motor cortical plasticity, Cereb. Cortex 23 (2013) 1593-1605.

[57] M.R. Goldsworthy, F. Müller-Dahlhaus, M.C. Ridding, U. Ziemann, Resistant agains de-depression: LTD-like plasticity in the human motor cortex induced by Spaced cTBS, Cereb. Cortex 25 (2015) 1724-1734.

[58] M.R. Goldsworthy, B. Hordacre, M.C. Ridding, Minimum number of trials required for within- and between-session reliability of TMS measures of corticospinal excitability, Neuroscience 320 (2016) 205-209.

[59] T. Chew, K.A. Ho, C.K. Loo, Inter- and intra-individual variability in response to transcranial direct current stimulation (tDCS) at varying current intensities, Brain Stimul. 8 (2015) 1130-1137.

[60] C. Ammann, M.A. Lindquist, P.A. Celnik, Response variability of different anodal transcranial direct current stimulation intensities across multiple sessions, Brain Stimul. 10 (2017) 757-763.

[61] S. Tremblay, F. Larochelle-Brunet, L.P. Lafleur, S. El Mouderrib, J.F. Lepage, $\mathrm{H}$. Theoret, Systematic assessment of duration and intensity of anodal transcranial direct current stimulation on primary motor cortex excitability, Eur. J. Neurosci. 44 (2016) 2184-2190

[62] J.C. Horvath, S.J. Vogrin, O. Carter, M.J. Cook, J.D. Forte, Effects of a common transcranial direct current stimulation (tDCS) protocol on motor evoked potentials found to be highly variable within individuals over 9 testing sessions, Exp. Brain Res. 234 (2016) 2629-2642.

[63] V. López-Alonso, M. Fernandez-Del-Olmo, A. Costantini, J.J. Gonzalez-Henriquez, B. Cheeran, Intra-individual variability in the response to anodal transcranial direct current stimulation, Clin. Neurophysiol. 126 (2015) 2342-2347.

[64] A. Jamil, G. Batsikadze, H.I. Kuo, L. Labruna, A. Hasan, W. Paulus, M.A. Nitsche, Systematic evaluation of the impact of stimulation intensity on neuroplastic aftereffects induced by transcranial direct current stimulation, J. Physiol 595 (2017) 1273-1288.

[65] S. Madhavan, A. Sriraman, S. Freels, Reliability and variability of tDCS induced 
changes in the lower limb motor cortex, Brain Sci. 6 (2016) (pii:E26).

[66] R. Puri, M.R. Hinder, A.J. Canty, J.J. Summers, Facilitatory non-invasive brain stimulation in older adults: the effect of stimulation type and duration on the in duction of motor cortex plasticity, Exp. Brain Res. 234 (2016) 3411-3423.

[67] K. Dyke, S. Kim, G.M. Jackson, S.R. Jackson, Intra-Subject consistency and reliability of response following $2 \mathrm{~mA}$ transcranial direct current stimulation, Brain Stimul. 9 (2016) 819-825.

[68] A.M. Vallence, M.R. Goldsworthy, N.A. Hodyl, J.G. Semmler, J.B. Pitcher, M.C. Ridding, Inter- and intra-subject variability of motor cortex plasticity following continuous theta-burst stimulation, Neuroscience 304 (2015) 266-278.

[69] F. Fratello, D. Veniero, G. Curcio, M. Ferrara, C. Marzano, F. Moroni, M.C. Pellicciari, M. Bertini, P.M. Rossini, L. De Gennaro, Modulation of corticospinal excitability by paired associative stimulation: reproducibility of effects and intraindividual reliability, Clin. Neurophysiol. 117 (2006) 2667-2674.

[70] D. Spampinato, P. Celnik, Temporal dynamics of cerebellar and motor cortex physiological processes during motor skill learning, Sci. Rep. 7 (2017) 40715.

[71] W. Strube, T. Bunse, M.A. Nitsche, A. Nikolaeva, U. Palm, F. Padberg, P. Falkai, A. Hasan, Bidirectional variability in motor cortex excitability modulation following $1 \mathrm{~mA}$ transcranial direct current stimulation in healthy participants, Physiol. Rep. 4 (2016) (pii:e12884).

[72] W. Strube, T. Bunse, B. Malchow, A. Hasan, Efficacy and interindividual variability in motor-cortex plasticity following anodal tDCS and paired-associative stimulation, Neural Plast. 2015 (2015) 530423.

[73] B. Hordacre, B. Moezzi, M.R. Goldsworthy, N.C. Rogasch, L.J. Graetz, M.C. Ridding, Resting state functional connectivity measures correlate with the response to anodal transcranial direct current stimulation, Eur. J. Neurosci. 45 (2017) 837-845.

[74] S. Wiethoff, M. Hamada, J.C. Rothwell, Variability in response to transcranial direct current stimulation of the motor cortex, Brain Stimul. 7 (2014) 468-475.

[75] T.W. Davidson, M. Bolic, F. Tremblay, Predicting modulation in corticomotor excitability and in transcallosal inhibition in response to anodal transcranial direct current stimulation, Front. Hum. Neurosci. 10 (2016) 49.

[76] D.S. Dickins, M.V. Sale, M.R. Kamke, Plasticity induced by intermittent theta burst stimulation in bilateral motor cortices is not altered in older adults, Neural Plast.
2015 (2015) 323409.

[77] M. Young-Bernier, A.N. Tanguay, P.S. Davidson, F. Tremblay, Short-latency afferent inhibition is a poor predictor of individual susceptibility to rTMS-induced plasticity in the motor cortex of young and older adults, Front. Aging Neurosci. 6 (2014) 182.

[78] C. Nettekoven, L.J. Volz, M. Leimbach, E.M. Pool, A.K. Rehme, S.B. Eickhoff, G.R. Fink, C. Grefkes, Inter-individual variability in cortical excitability and motor network connectivity following multiple blocks of rTMS, Neuroimage 118 (2015) 209-218.

[79] M.J. Player, J.L. Taylor, A. Alonzo, C.K. Loo, Paired associative stimulation increases motor cortex excitability more effectively than theta-burst stimulation, Clin. Neurophysiol. 123 (2012) 2220-2226.

[80] B. Hordacre, M.R. Goldsworthy, A.M. Vallence, S. Darvishi, B. Moezzi, M. Hamada, J.C. Rothwell, M.C. Ridding, Variability in neural excitability and plasticity induction in the human cortex: a brain stimulation study, Brain. Stimul. 10 (2017) 588-595.

[81] T. Heidegger, O. Hansen-Goos, O. Batlaeva, O. Annak, U. Ziemann, J. Lötsch, A data-driven approach to responder subgroup identification after paired continuous theta burst stimulation, Front. Hum. Neurosci. 11 (2017) 382.

[82] C.E. Palmer, K.L. Bunday, M. Davare, J.M. Kilner, A causal role for primary motor cortex in perception of observed actions, J. Cogn. Neurosci. 28 (2016) 2021-2029.

[83] A. Jannati, G. Block, L. Oberman, A. Rotenberg, A. Pascual-Leone, Interindividual variability in response to continuous theta-burst stimulation (cTBS) in healthy adults, Clin. Neurophysiol. 128 (2017) 2268-2278.

[84] G. Huang, A. Mouraux, MEP latencies predict the neuromodulatory effect of cTBS delivered to the ipsilateral and contralateral sensorimotor cortex, PLoS One 10 (2015) e0133893.

[85] J. Lahr, S. Passmann, J. List, W. Vach, A. Floel, S. Kloppel, Effects of different analysis strategies on paired associative stimulation. A pooled data analysis from three research labs, PLoS One 11 (2016) e0154880.

[86] J.F. Muller-Dahlhaus, Y. Orekhov, Y. Liu, U. Ziemann, Interindividual variability and age-dependency of motor cortical plasticity induced by paired associative stimulation, Exp. Brain Res. 187 (2008) 467-475. 